\title{
Mast Cell and M1 Macrophage Infiltration and Local Pro-Inflammatory Factors Were Attenuated with Incretin-Based Therapies in Obesity-Related Glomerulopathy
}

\author{
Jiao He, PhD, Geheng Yuan, PhD, Fangxiao Cheng, PhD, Junqing Zhang, PhD, and Xiaohui Guo, PhD
}

\begin{abstract}
Background: The global increase of obesity parallels the obesity-related glomerulopathy (ORG) epidemic. Dipeptidyl peptidase 4 inhibitors and glucagon-like peptide-1 receptor agonists were well recognized to attenuate renal injury independent of glucose control in diabetic nephropathy. There are limited studies focusing on their effects on ORG. We explored the effects of incretin-based therapies on early ORG and the inflammatory responses involved mainly concentrated on mast cell (MC) and macrophage (M) infiltration and local pro-inflammatory factors.

Methods: ORG rat models were induced by high-fat diet and then divided into ORG vehicle, vildagliptin ( $3 \mathrm{mg} /$ $\mathrm{kg} / \mathrm{day}, q d)$ and liraglutide $(200 \mu \mathrm{g} / \mathrm{kg}$, bid $)$ treated groups. After 8 weeks of treatments, albuminuria, glomerular histology, renal inflammatory cell infiltration, and pro-inflammatory factors were analyzed.

Results: Early ORG model was demonstrated by albuminuria, glomerulomegaly, foot process fusion, and mesangial and endothelial mild proliferation. Incretin-based therapies limited body weight gain and improved insulin sensitivity. ORG was alleviated, manifested by decreased average glomerular area, attenuated mesangial and endothelial cell proliferation, and revived cell-to-cell propagation of podocytes, which contributed to reduced albuminuria. Compared with ORG vehicle, MC and M1 macrophage (pro-inflammatory) infiltration and M1/M2 ratio were significantly decreased; M2 macrophage (anti-inflammatory) was not significantly increased after incretin-based treatments. Tumor necrosis factor- $\alpha$ (TNF- $\alpha$ ) and IL- 6 in renal cortex were significantly downregulated, while transforming growth factor- $\beta 1$ (TGF- $\beta 1$ ) remained unchanged.

Conclusions: Incretin-based treatments could alleviate high-fat diet-induced ORG partly through the systemic insulin sensitivity improvement and the attenuated local inflammation, mainly by the decrease of MC and M1 macrophage infiltration and reduction of TNF- $\alpha$ and IL- 6 .
\end{abstract}

Keywords: obesity-related glomerulopathy, incretin, DPP4, mast cell, macrophage

\section{Introduction}

\footnotetext{
I
} $\mathrm{N}$ THE LAST THREE DECADES, the proportion of overweight and obese adults has increased from $28.8 \%$ to $36.9 \%$ among men and from $29.8 \%$ to $38.0 \%$ among women worldwide. ${ }^{1}$ A less investigated complication of obesity is obesityrelated glomerulopathy (ORG). Early changes occurring in ORG include glomerular hyperfiltration, glomerulomegaly, mesangial expansion, and foot process effacement-induced albuminuria, leading to focal segmental glomerulosclerosis. ${ }^{2-5}$
In experimental studies, various mechanisms have been propounded for ORG, such as lipotoxicity-related endothelial dysfunction, oxidative stress, and persistent inflammation. ${ }^{6}$

Obesity is recognized as an inflammatory disorder. This inflammatory response is characterized by the recruitment of inflammatory cells, their interaction with resident kidney cells, and the generation of inflammatory mediators to counteract disease. Macrophages are well documented for participating in various inflammatory responses. Macrophages polarize into classical M1 or alternative M2. Once

Department of Endocrinology, Peking University First Hospital, Beijing, People's Republic of China.

(C) Jiao He et al. 2017; Published by Mary Ann Liebert, Inc. This article is available under the Creative Commons License CC-BY-NC (http://creativecommons.org/licenses/by-nc/4.0). This license permits non-commercial use, distribution and reproduction in any medium, provided the original work is properly cited. Permission only needs to be obtained for commercial use and can be done via RightsLink. 
activated, M1 macrophages release tumor necrosis factor- $\alpha$ (TNF- $\alpha$ ), interleukin-6 (IL-6), and monocyte hemoattractant protein (MCP-1), which underlie the pathology of renal injury. M2 macrophages express CD206 and participate in anti-inflammation, tissue repair, or remodeling. ${ }^{7-9}$ Mast cells (MCs) are bone marrow-derived cells, ${ }^{10}$ which are well known for their roles in anaphylactic reactions. MC degranulation extrudes numerous mediators such as chymase, tryptase, histamine, and renin ${ }^{11-13}$ and cytokines such as TNF- $\alpha$, vascular endothelial growth factor, and transforming growth factor- $\beta$ (TGF- $\beta$ ). ${ }^{14}$ Furthermore, MC infiltration has proven to be a prominent and early feature following renal injury; an increase of MCs is associated with fibrosis and extracellular matrix accumulation in the kidneys. ${ }^{15,16}$ Wang et al. reported that $\mathrm{MC}$ infiltration is involved in renal damage of ORG patients. ${ }^{16}$ Treatment with MC stabilizers serves as potential therapy in management of high-fat diet (HFD)induced renal dysfunction in rats. ${ }^{17}$ It is suggested that a pharmacotherapy might be needed in ORG to inhibit MC infiltration, decrease inflammation, and reduce weight.

Incretin-based therapies, glucagon-like peptide-1 receptor agonist (GLP-1RA), and dipeptidyl peptidase 4 inhibitor (DPP4) have been widely used in diabetes area. A reduction of albuminuria and an improvement in histological changes in the kidney were observed in type 1 diabetes mellitus (T1DM) rat model treated with vildagliptin $(\mathrm{VG}){ }^{18}$ In the experimental model of renal ischemia/reperfusion injury treated with $\mathrm{VG}$, DPP4 inhibition produced renoprotective effects that were mediated by antiapoptotic, anti-inflammatory, and antioxidative effects. ${ }^{19}$ In the Liraglutide Effect and Action in Diabetes: Evaluation of Cardiovascular Outcome Results (LEADER) study, there were renoprotective effects of liraglutide (LG) to reduce macroalbuminuria in type 2 diabetes mellitus (T2DM) patients. ${ }^{20}$ However, only a few studies have reported the effects of incretin-based therapies on ORG model.

In the present study, we investigated the potential role of DPP4i and GLP-1RA in ORG, including the efficacy (albuminuria etc.) and effects on inflammation, especially MC and macrophage infiltration and local pro-inflammatory mediators in early ORG rat model.

\section{Materials and Methods}

\section{Animals and experimental procedures}

All of the experimental procedures were approved by the Animal Experiments Ethics Committee of Peking University First Hospital. Six-week-old male Sprague Dawley rats were purchased from Beijing Vital River Laboratory
Animal Technology Co. Ltd. They were kept in the Laboratory Animal Center of Peking University First Hospital with 12-hr light to dark cycles at $22^{\circ} \mathrm{C} \pm 2{ }^{\circ} \mathrm{C}$.

Rats were divided into two groups after 1-week adaption; one group $(n=10)$ received normal diet $(\mathrm{ND}, 10 \% \mathrm{kcal}$ from fat), as lean control (Con); the other $(n=22)$ received HFD (D12492, 60\% kcal from fat) to induce obese rat model, as obese group; both were ad libitum with free access to water.

When body weight was significantly increased and albuminuria was detected positive in the obese group for about 12 weeks, we evaluated insulin sensitivity. At the end of 14th week, five control rats and five obese rats were sacrificed. We conducted histopathologic examinations to further estimate ORG model. The rest obese rats with continued HFD were further randomized into ORG vehicle, ORG treated with VG (3 mg/kg/day, $q d$, intragastric infusion) and ORG treated with LG $(200 \mu \mathrm{g} / \mathrm{kg}$, bid, subcutaneous injection) groups. Body weights were measured every week. Fasting blood glucose (FBG) and fasting insulin (FINS) were measured before and after treatments. Homeostasis model assessment of insulin resistance (HOMA-IR) was calculated according to the formula: HOMA-IR $=$ FBG $\times$ FINS/22.5. After 8 weeks of intervention, all rats were sacrificed (Fig. 1).

\section{Reagents}

All of the primary antibodies were purchased from Abcam plc (Cambridge, United Kingdom), including MC tryptase, CD68, mannose receptor (CD206), TNF- $\alpha$, IL-6, and TGF$\beta 1$. Tryptase, derived from MC degranulation, serves as MC specific marker. CD68 and CD206, located in biological membrane, serve as specific markers of M1 macrophage and M2 macrophage, respectively.

\section{Albuminuria measurements}

Both albumin $(\mathrm{Ab})$ and creatinine $(\mathrm{Cr})$ concentrations in urine were analyzed using commercially available ELISA Kit (Exocell, Inc., Philadelphia, PA) according to the instructions.

\section{Oral glucose tolerance test}

Oral glucose tolerance test (OGTT) was performed before and after intervention. Rats were fasted for $12 \mathrm{hr}$. Oral glucose load was administered using gavage at $2 \mathrm{~g} / \mathrm{kg}$ body weight. Blood glucose levels were measured from caudal vein using Glucometer Elite (Roche Corp., Basel, $\mathrm{CH}$ ) at 0 , 30, 60, 90, 120 (and 180) minutes (min).

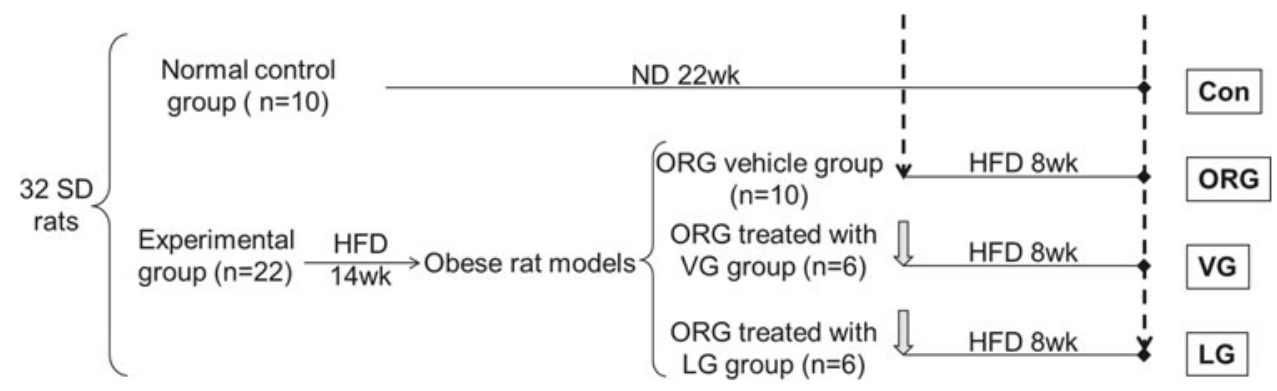

FIG. 1. Experimental procedure. Dashed arrow represents the time point of sacrifice. Gray solid thick arrow represents the start point of drug administration. Solid dot represents the end of experiment. Con, control; HFD, high-fat diet; LG, liraglutide; ND, normal diet; ORG, obesity-related glomerulopathy; SD, Sprague Dawley; VG, vildagliptin. 
Table 1. Sequences of Primers Used for Real-Time Polymerase Chain Reaction (Rat)

\begin{tabular}{|c|c|c|}
\hline \multirow[b]{2}{*}{ Target gene } & \multicolumn{2}{|c|}{ Sequence } \\
\hline & Forward & Reverse \\
\hline$T N F-\alpha$ & 5'-TGGGCTCCСТCTCATCAGTT-3' & 5'-GCTTGTCACTCGAGTTTTGAGAAG-3' \\
\hline$I L-6$ & 5'-CAGAGGATACCACCCACAACAG-3' & $5^{\prime}$-TCAGAATTGCCATTGCACAAC- $3^{\prime}$ \\
\hline$T G F-\beta 1$ & 5'-GGAGAGCCCTGGATACCAACT-3' & 5'-TCCAACCCAGGTCCTTCCTA-3' \\
\hline$G A P D H$ & 5'-TGCACCACCAACTGCTTAGC-3' & 5'-GCAGTGATGGCATGGACTGT-3' \\
\hline
\end{tabular}

GAPDH, glyceraldehyde-phosphate dehydrogenase; IL- 6 , interleukin-6; TGF- $\beta 1$, transforming growth factor- $\beta 1$; TNF- $\alpha$, tumor necrosis factor- $\alpha$.

A
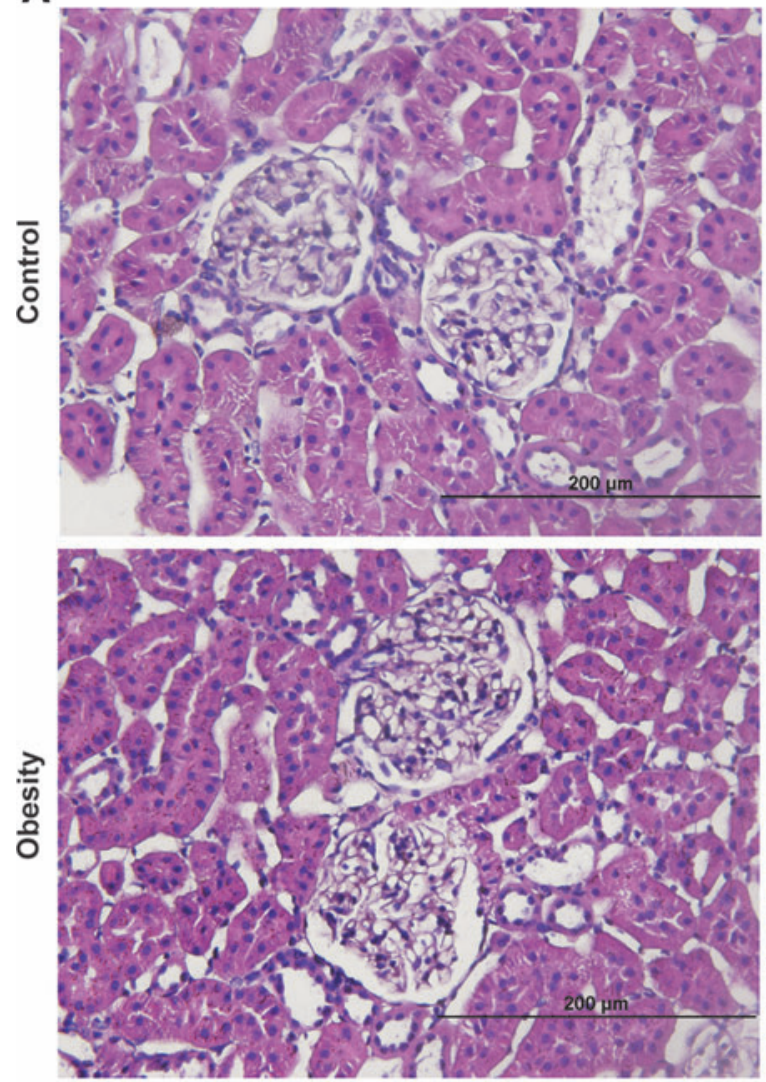

B

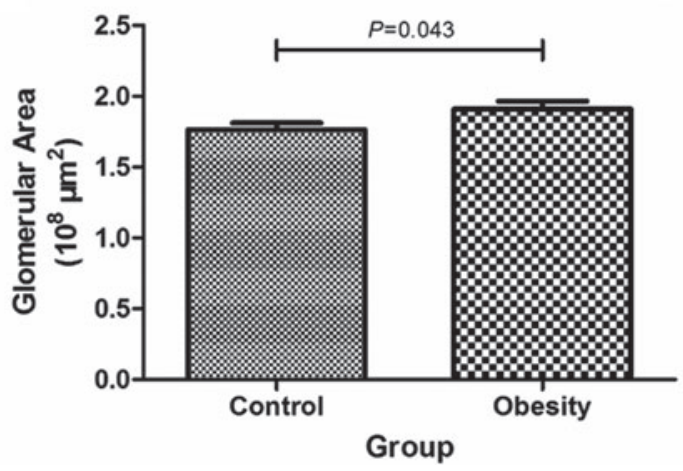

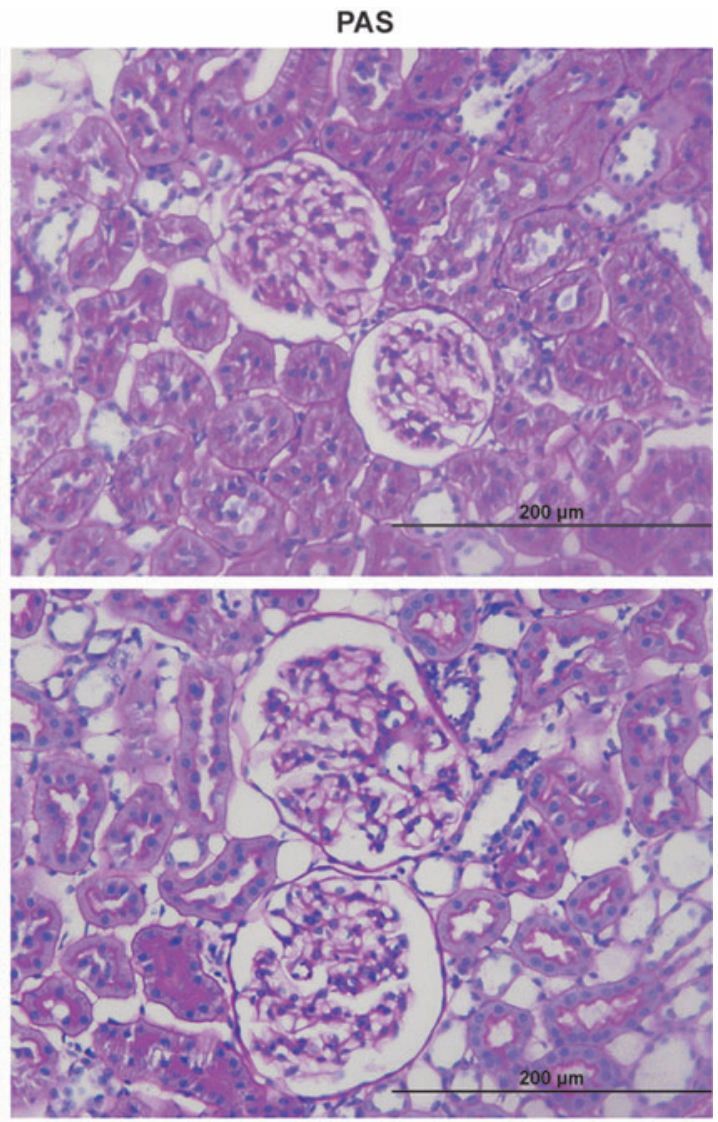

C

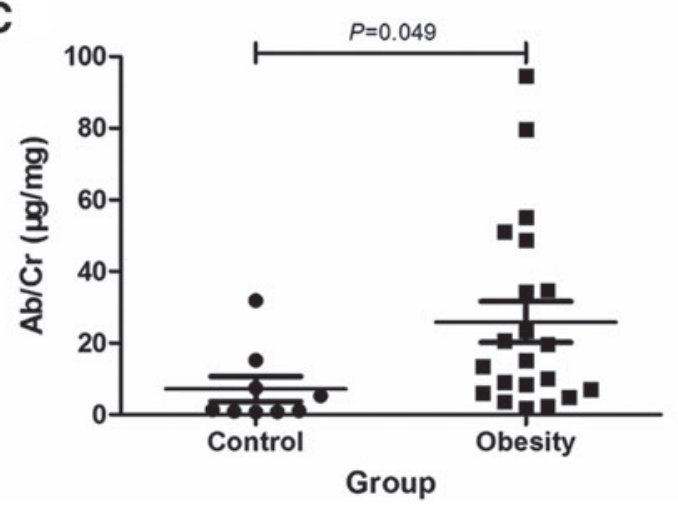

FIG. 2. Representative photomicrographs of HE and PAS staining from renal cortex sections, glomerular area, and albuminuria analyses. (A) Glomerular histology of HE and PAS staining (magnification $400 \times$ ). (B) Average glomerular area of the obese group was significantly larger than that in the lean control group. Control, $n=5$; obesity, $n=5$. (C) The ratio of $\mathrm{Ab}$ to $\mathrm{Cr}$ in the obese group was significantly higher compared with the lean control group. Control, $n=9$; obesity, $n=21$. HE, hematoxylin and eosin; PAS, periodic acid-Schiff; Ab, abumin; Cr, creatinine. 


\section{Intraperitoneal insulin tolerance test}

Intraperitoneal insulin tolerance test (IPITT) was performed the same as OGTT except the insulin load was administered through intraperitoneal injection at $0.75 \mathrm{IU} / \mathrm{kg}$ body weight.

\section{Histopathologic evaluation}

Kidney cortex was fixed in $10 \%$ neutral buffered formalin and embedded in paraffin for hematoxylin and eosin (HE), and periodic acid-Schiff (PAS) staining, fixed in 1\% glutaraldehyde to make transmission electron microscope (TEM) semi-thin sections. Paraffin sections were examined under a light microscope, and the images were captured using DPC Controller and Manager softwares (OLYMPUS Co., Tokyo, Japan). The glomerular area was acquired in five randomly selected nonoverlapping fields $(200 \times)$ per rat with HE stained kidney tissues ${ }^{21}$ and then was quantified by ImageJ software (National Institutes of Health, Bethesda, MD). PAS staining was used to evaluate mesangial cell proliferation and mesangial matrix expansion. TEM sections were estimated for glomerular ultrastructure.

\section{Immunohistochemistry}

After deparaffinization and rehydration, renal cortex tissue sections $(4 \mu \mathrm{m})$ underwent endogenous peroxidase activity inhibition and antigen retrieval in microwave. Slides were incubated with confining liquid for $30 \mathrm{~min}$ at room temperature (RT) to wipe off background coloration; incubated with primary antibodies (anti-MC tryptase, antiCD68, or anti-CD206) overnight at $4^{\circ} \mathrm{C}$; and then incubated with secondary horseradish peroxidase (HRP)-conjugated antibody (Beijing Zhongshan Golden Bridge Biotechnology Co., Beijing, CN) for $1 \mathrm{hr}$ at RT. After staining the nucleus with hematoxylin, sections were incubated with diaminobenzidine (DAB) for coloration. Images were recorded using DPC Controller and Manager softwares. A total of 48 fields of renal cortex in each section were examined in a sequence of adjacent fields at a magnification of $400 \times$, avoiding glomeruli or major vessels. ${ }^{16,22}$ The positive cell numbers were quantified by counting brown cells per field.

\section{Western blot analysis}

Renal cortex total protein was extracted and quantified using a Bicinchoninic Acid (BCA) Protein Quantification Kit
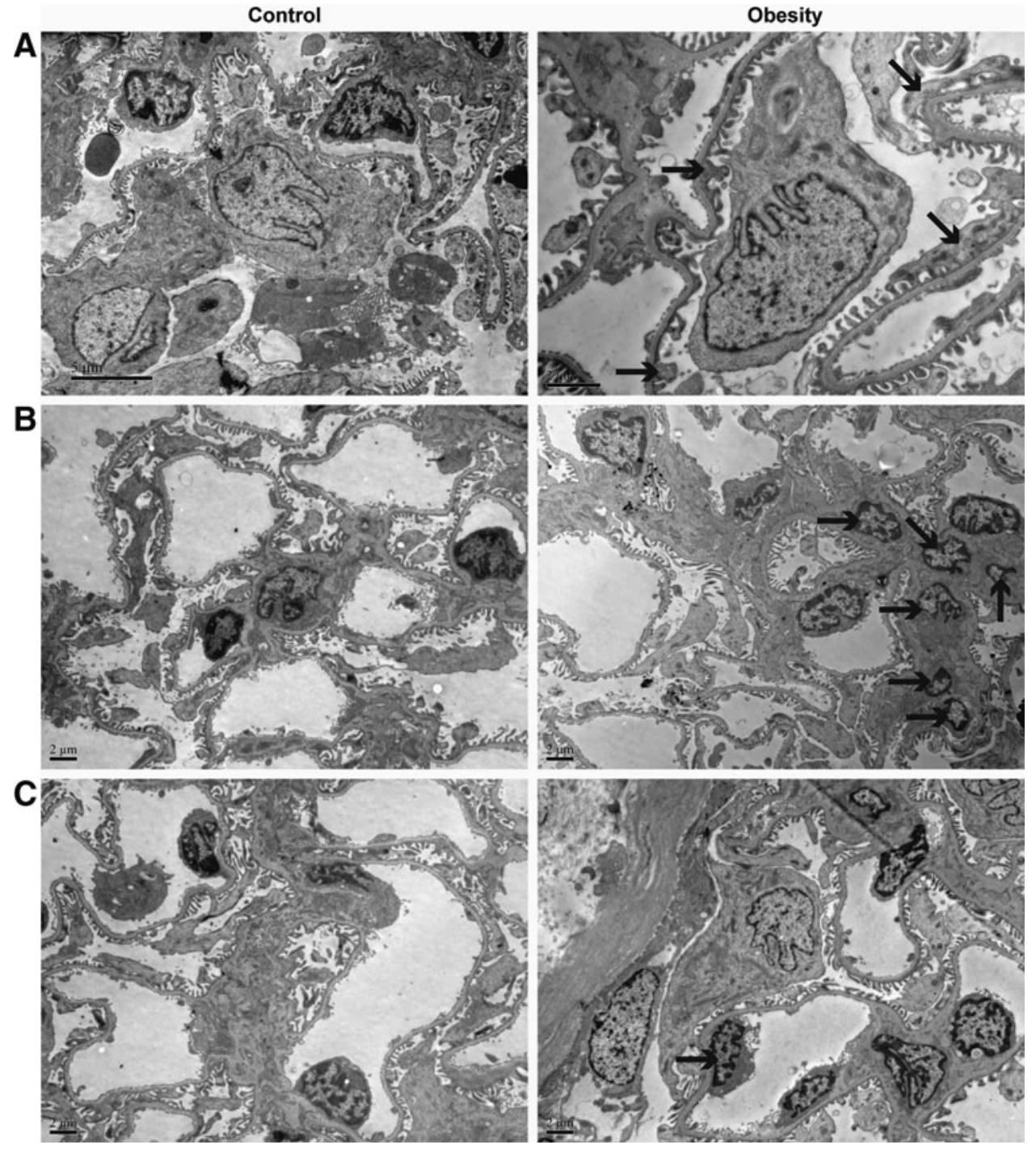

FIG. 3. Representative photomicrographs of transmission electron microscope from renal cortex semi-thin sections. In the obese group, renal injury was observed, demonstrated by (A) foot process fusion (B) mesangial cell mild proliferation and mesangial matrix expansion $(\mathbf{C})$ capillary EC mild proliferation. Black solid arrow points to fusional foot processes in (A), proliferative mesangial cells in (B), and proliferative ECs in (C). ECs, endothelial cells. 
(Thermo Fisher Scientific Co., MA). The same amount of total protein from each sample was separated by sodium dodecyl sulfate-polyacrylamide gel electrophoresis (SDSPAGE) and transferred to a nitrocellulose membrane $(0.45 \mu \mathrm{m}$ pore size; PALL Corporation, Port Washington, NY). The membranes were blocked in confining liquid for $1 \mathrm{hr}$ at RT; incubated with primary antibodies at $4{ }^{\circ} \mathrm{C}$ overnight; and then incubated with secondary HRP-conjugated antibody for $1 \mathrm{hr}$ at RT. The membranes were visualized with enhanced chemiluminescence reagents (EMD Millipore, Billerica, MA).

\section{Real-time polymerase chain reaction}

Renal cortex total RNA was isolated using TRIzol reagent. First-strand cDNA was synthesized from total RNA using a reverse transcriptase synthesis system (SuperScript III; Invitrogen, Carlsbad, CA) and random hexamers. Real- time polymerase chain reaction (RT-PCR) was performed with a sequence detection system (ABI Prism 7300; Thermo Fisher Scientific Co.). The messenger RNA (mRNA) levels were calculated by comparative $\mathrm{Ct}$ methods $\left(\mathrm{X}_{\text {Test }} /\right.$ $\mathrm{X}_{G A P D H}=2^{-\triangle \Delta \mathrm{Ct}}$ ) with $G A P D H$ as the endogenous reference gene. Primers and probes were designed by Primer Express 3.0.1 (Thermo Fisher Scientific Co.) (Table 1).

\section{Statistical analysis}

All values are presented as mean \pm standard error of mean. The statistical significance of the difference was analyzed by unpaired two-tailed $t$-test between two groups or one-way analysis of variance when more than two groups, followed by Tukey's multiple comparison test using GraphPad Prism software (GraphPad Software, Inc., CA). The trapezoidal rule was applied to analyze the area under the curve (AUC). A

A
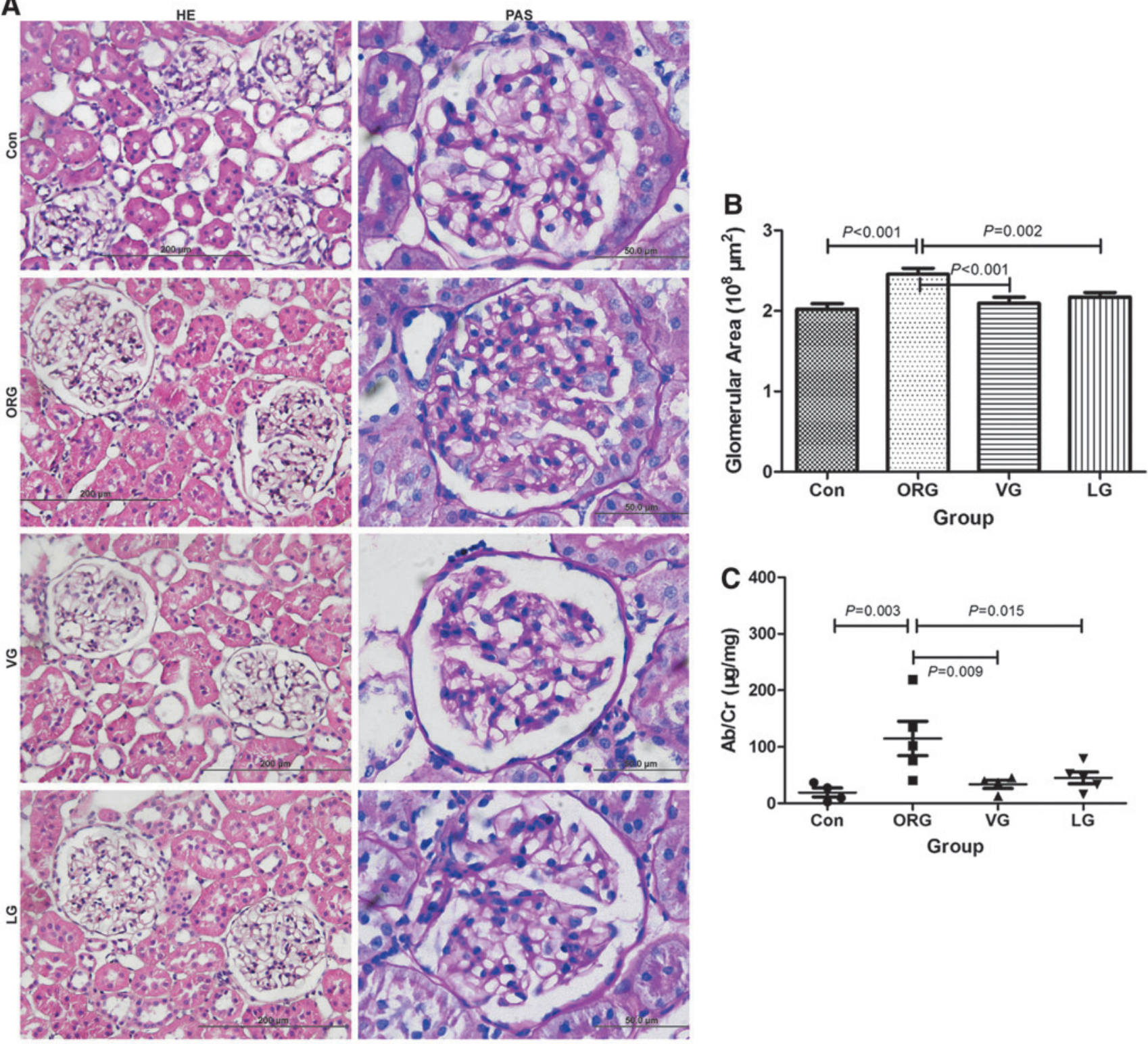

FIG. 4. Effects of incretin-based treatments on glomerular histology, average glomerular area, and albuminuria of ORG rats.

(A) Representative photomicrographs (HE, magnification $\times 400$; PAS, magnification $\times 1000$ ) of glomerular histology. (B) Average glomerular area was significantly reduced compared with $\mathrm{ORG}$ vehicle group. (C) $\mathrm{The}$ ratio of $\mathrm{Ab}$ to $\mathrm{Cr}$ was significantly decreased after VG and LG treatments. $n=4$ in each group. 
value of $P<0.05$ was accepted as statistical significance. The data obtained were illustrated using GraphPad Prism software or Adobe Illustrator CS5 softwares.

\section{Results}

\section{HFD induced ORG rat model}

Rats were fed with HFD to induce ORG model (Supplementary Table S1; Supplementary Data are available online at www.liebertpub.com/met). After 14 weeks, the body weight, FINS, and HOMA-IR were significantly increased in obese group. Compared with lean control group, AUC of glucose in OGTT was significantly increased, whereas FBG was not significantly different (Supplementary Figs. S1 and S2). It was not in accordance with diagnostic criteria of diabetes. Obesity-induced renal injury was observed, demonstrated by albuminuria (Fig. 2C), glomerular hypertrophy (Fig. 2A, B), mesangial cell and capillary endothelial cell (EC) proliferation, as well as foot process fusion or effacement (Fig. 3). There was no significant difference of glomerular basement membrane thickness between the two groups.

\section{Incretin-based treatments limited body weight gain and improved insulin sensitivity}

After 8 weeks of intervention, the body weight gain of both VG and LG groups was significantly lower than that in ORG vehicle group; there was no significant difference between two incretin therapies (Supplementary Fig. S3). After treatments, FBG, FINS, and HOMA-IR were not significantly reduced. The AUC of glucose in IPITT was significantly reduced in LG group $(P=0.021)$, while AUC of glucose in OGTT was not significantly reduced in both treatment groups (Supplementary Fig. S4 and Supplementary Table S2).

\section{Incretin-based treatments improved HFD-induced ORG}

Average glomerular area was significantly reduced and albuminuria was significantly attenuated after VG and LG treatments; there was no significant difference between two incretin therapies (Fig. 4). The glomerular injury was also ameliorated, including revived cell-to-cell propagation of podocyte, decreased mesangial cell numbers, reduced mesangial area, and less common EC proliferation (Figs. 4 and 5).
A
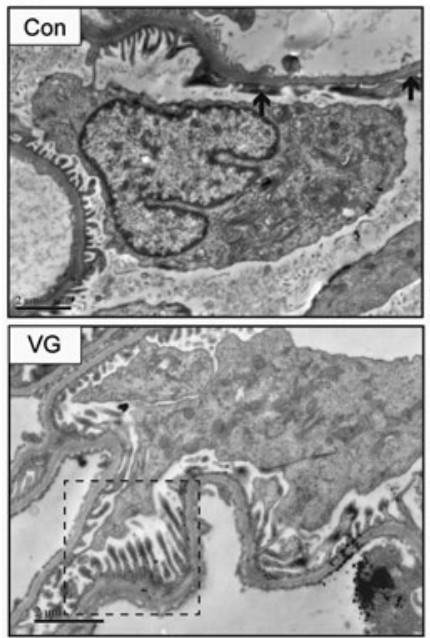
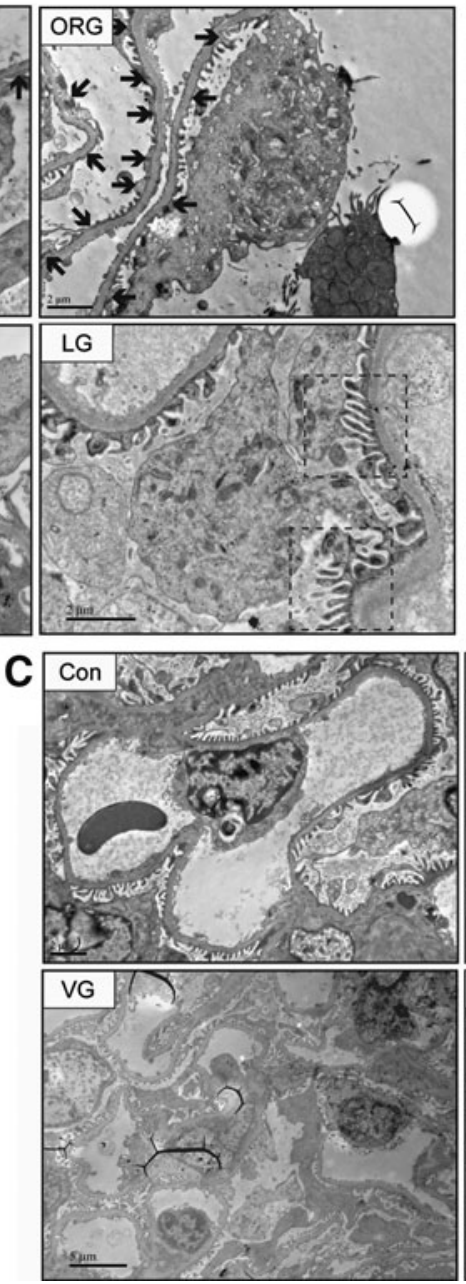

B
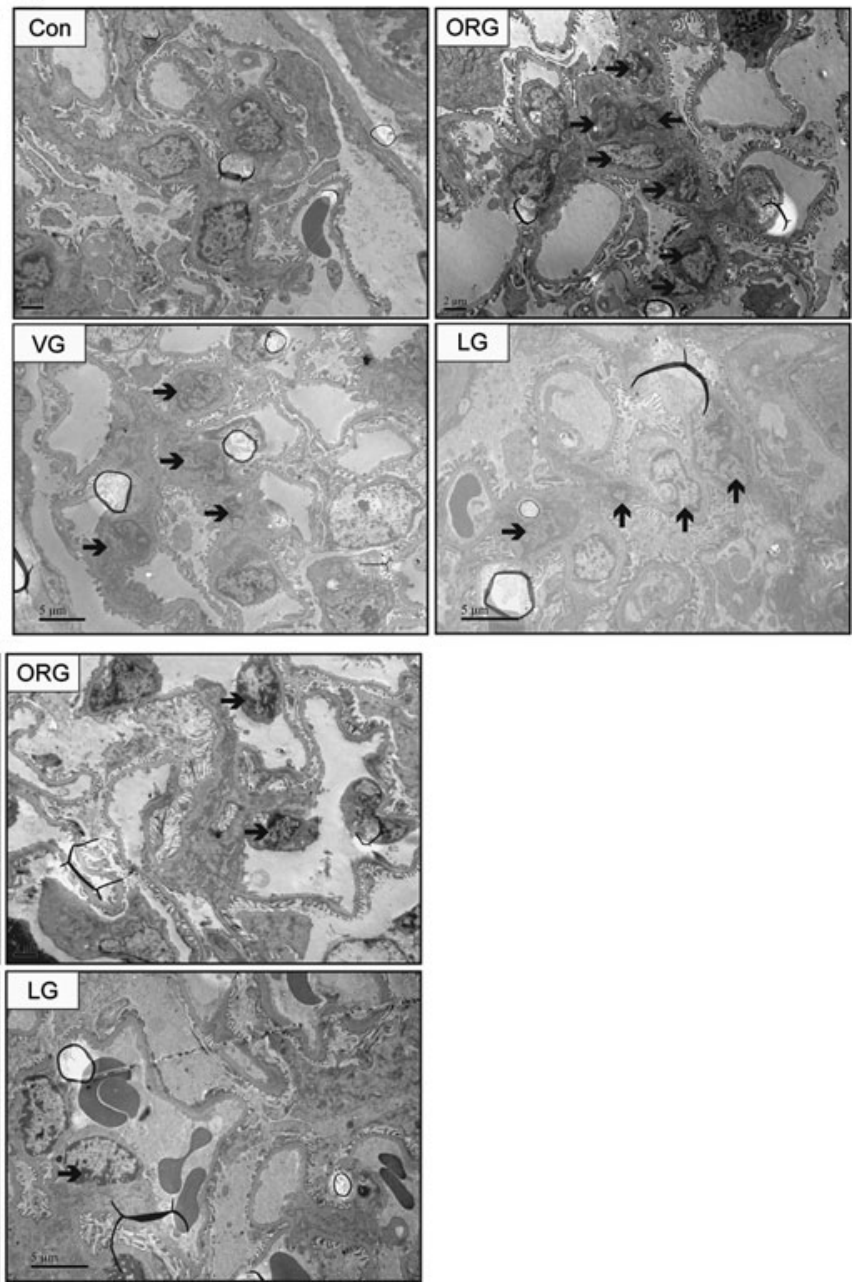

FIG. 5. Effects of incretin-based treatments on glomerular ultrastructure of ORG rats. After VG and LG treatments (A) cell-tocell propagation of podocyte was partly revived; (B) mesangial cell proliferation was attenuated and mesangial area was reduced; and (C) capillary EC proliferation was alleviated. Black solid arrow points to fusional foot processes in (A), proliferative mesangial cells in (B), and proliferative ECs in (C). Black dotted box points to revived cell-to-cell propagation of podocytes. 


\section{Incretin-based treatments reduced $M C$ and $M 1$ macrophage numbers and decreased $M 1 / M 2$ ratio}

Renal MCs and macrophages were primarily resident in renal interstitium, but not within the glomeruli. MC and CD68positive M1 macrophage infiltration of ORG group were significantly increased compared with that in lean control and were significantly decreased after VG and LG treatments (Fig. 6A, B, D), while the number of CD206-positive M2 macrophages was fewer in the ORG group compared with lean control and was not significantly increased after treatments (Fig. 6C, D). The ratio of M1 to M2 was significantly increased in ORG vehicle group, which was significantly decreased after treatments (Fig. 6D and Supplementary Table S3).

\section{Incretin-based treatments reduced the level of pro-inflammatory factors}

TNF- $\alpha$ and IL-6 levels were significantly increased in ORG group compared with lean control. After VG and LG treatments for 8 weeks, TNF- $\alpha$ and IL-6 levels were significantly decreased (Fig. 7A, B, D, E). However, TGF- $\beta 1$, which is involved in interstitial fibrosis, was unchanged after treatments (Fig. 7C, F and Supplementary Table S4).

\section{Discussion}

In the present study, we have successfully created early ORG rat model, demonstrated by albuminuria, glomerular hypertrophy, foot process fusion or effacement, and capillary EC and mesangial cell mild proliferation. Incretin-based treatments could limit body weight gain, reduce albuminuria, alleviate foot process fusion, mesangial cell and EC proliferation, and decrease $\mathrm{MC}$, pro-inflammatory M1 macrophage infiltration, and local pro-inflammatory factors. To the best of our knowledge, we are the first ones to report the effects of incretin-based therapies on MCs in HFDinduced ORG.

Because it is an in vivo study, the phenotype amelioration may be ascribed to the systemic effects of incretin-based therapies first. Moderate weight loss in overweight patients with chronic proteinuric nephropathies induces a significant decrease in albuminuria. ${ }^{23} \mathrm{~A}$ favorable modulation of insulin resistance (IR) has a positive effect on urinary albumin
A

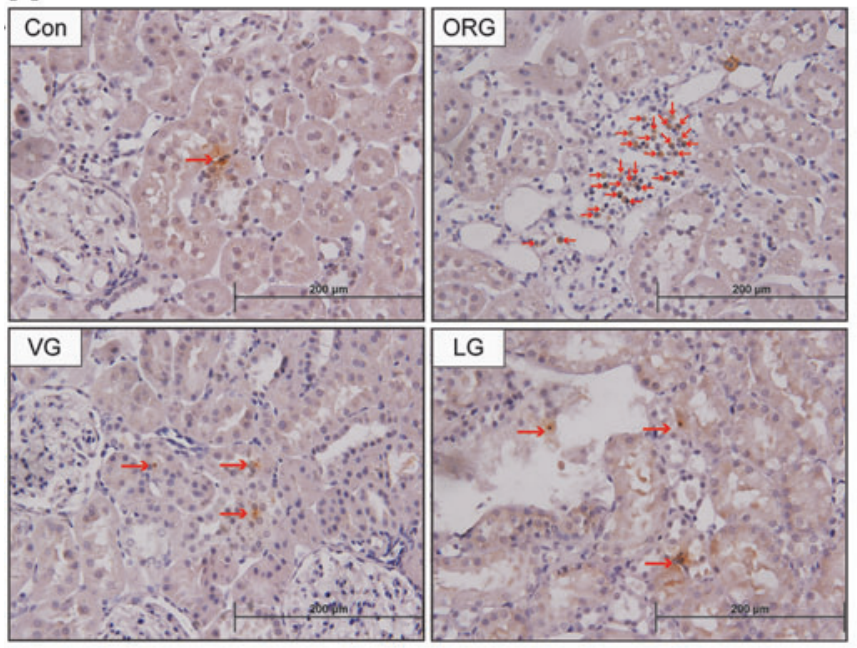

C
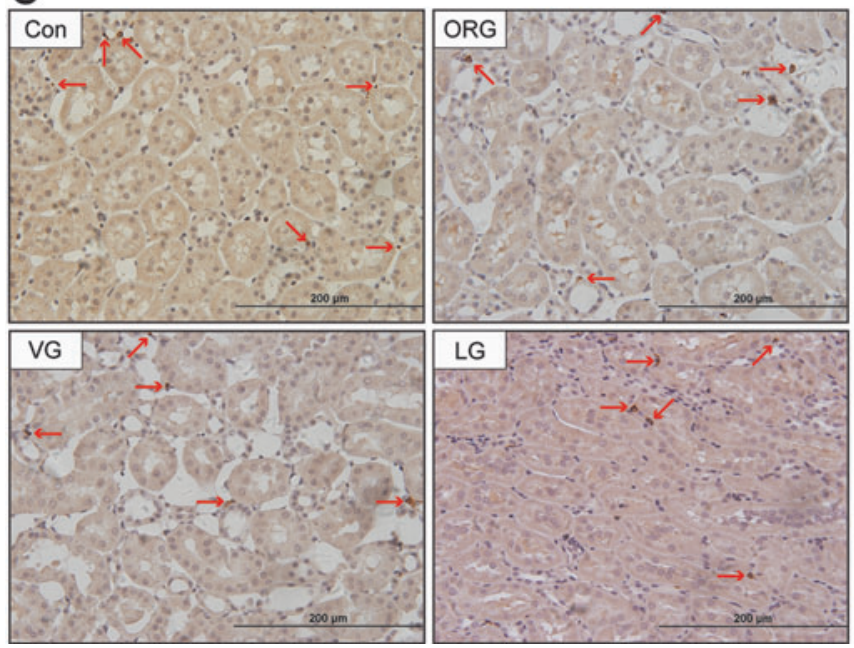

B

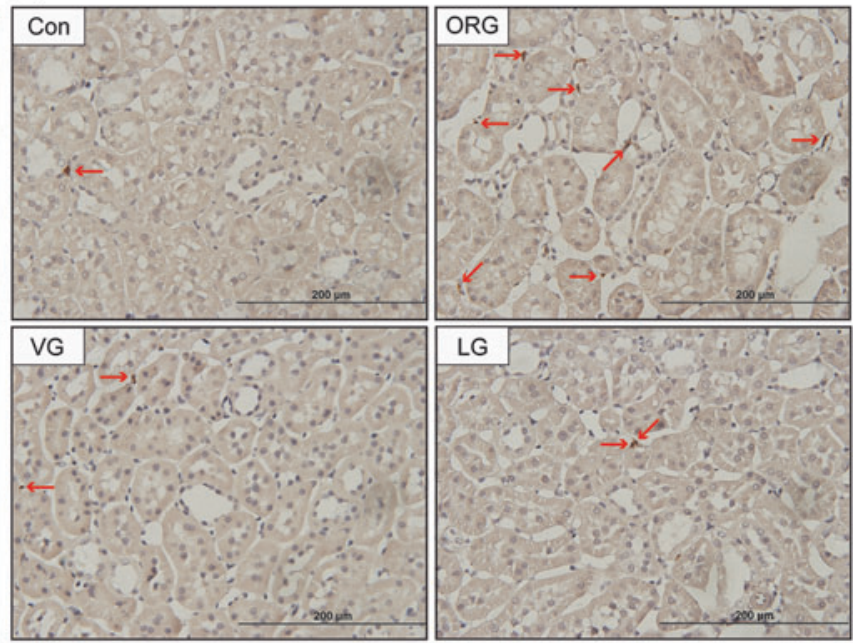

D
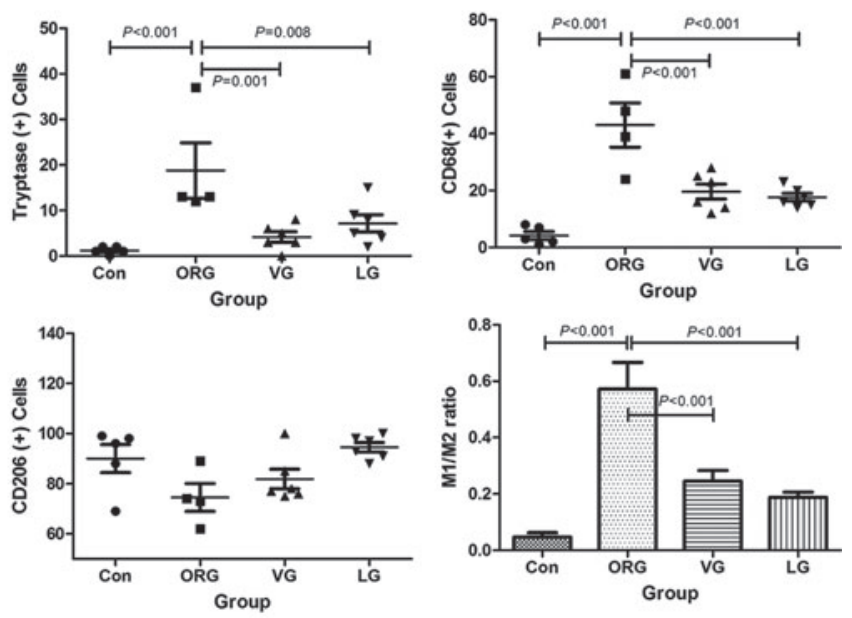

FIG. 6. Representative photomicrographs and analyses of tryptase, CD68, and CD206 immunohistochemistry in ORG rats after treatments. (A) MC infiltration. (B) M1 macrophage infiltration. (C) M2 macrophage infiltration. (D) Counts for MCs and macrophages and the relative ratio of M1 to M2. Red arrow points to positive cells. MC, mast cell. 

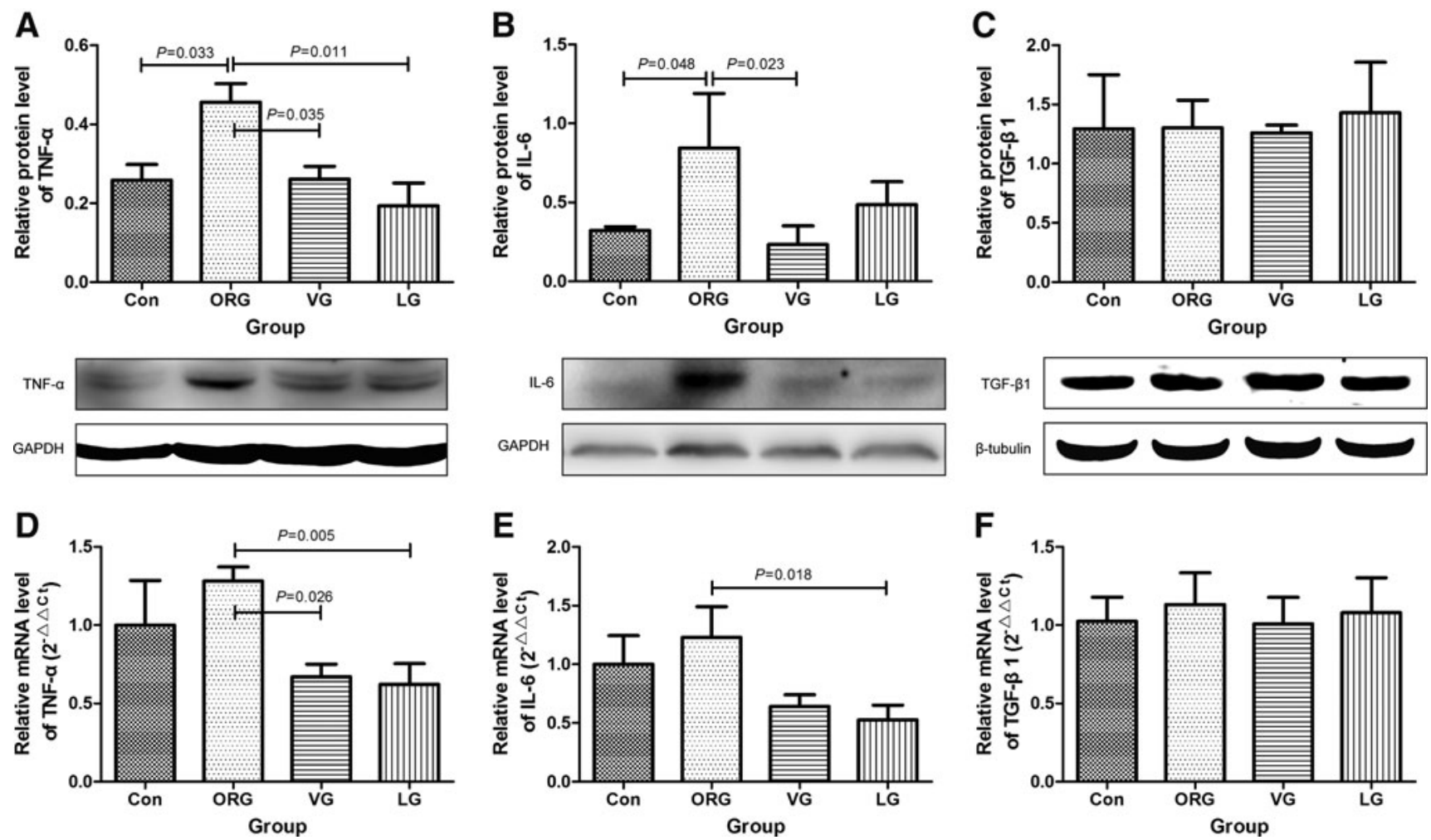

FIG. 7. Effects of incretin-based treatments on protein and mRNA levels of pro-inflammatory factors of ORG rats. (A-C) Quantification data with corresponding panels of Western blot. (D-F) mRNA expression by real-time polymerase chain reaction. In (A-F) $n=4$ in each group. In (C) $n=3$ in each group. mRNA, messenger ribonucleic acid. IL-6, interleukin-6; TGF- $\beta 1$, transforming growth factor- $\beta 1$; TNF- $\alpha$, tumor necrosis factor- $\alpha$.

excretion. ${ }^{24}$ In the present study, incretin-based treatments could limit body weight gain and ameliorate insulin sensitivity, and albuminuria was alleviated. More attention was paid to the local inflammation in ORG.

In our findings: (1) MCs and macrophages were mostly resident in the renal interstitium, but not within the glomeruli; (2) the density of MCs and M1 macrophages was paralleled with the extent of renal damage; (3) MCs in renal cortex were significantly decreased after incretin-based treatments. M1 macrophages (pro-inflammatory) were significantly decreased, while M2 macrophages (anti-inflammatory) were not significantly increased; the relative ratio of M1 to M2 was significantly decreased compared with vehicle control; (4) TNF- $\alpha$ and IL-6 in renal cortex were significantly decreased, but TGF- $\beta 1$ was the same among all groups.

Evidence showed that $\mathrm{MC}$ infiltration increases in ORG. ${ }^{16} \mathrm{MC}$ infiltration and the cytokines and chemokines they produce lead to progressive renal disease. ${ }^{25}$ After incretin-based therapies in the present study, the number of MCs was decreased and renal injury was alleviated. It was consistent with MC inactivation which was effective to treat inflammatory diseases in other renal injury animal models. ${ }^{26}$ But there was limited knowledge about MC-involved pathways. Gschwandtner et al. showed that DPP4 was found to be expressed in skin and bone marrow MCs. ${ }^{27}$ Thus VG may have direct effects on MCs. Besides, increment of specialized pro-resolving mediators (SPMs) may be another important factor. It is likely that SPMs inhibited MC infiltration in adipose tissue, and SPMs appeared deficient in obese visceral adipose tissue. ${ }^{28}$ It was demonstrated that exenatide increased myocardial lipoxins (a kind of SPMs) and attenuated inflammation in ob/ob mice. ${ }^{29}$ Further investigations should be taken on whether GLP-1-based therapies promote SPM production in ORG.

Increased macrophage infiltration was observed in ORG patients. ${ }^{16}$ Various studies have shown that GLP-1-based therapies can reduce macrophage infiltration in models of nondiabetic nephropathy. ${ }^{30}$ Higashijima et al. revealed that exendin-4, but not alogliptin, reduced MCP-1-stimulated macrophage infiltration in a dose-dependent manner in the rat Thy-1 nephritis model. It is suggested that DPP4 inhibitors may reduce macrophage infiltration through GLP-1dependent signaling. ${ }^{30}$

The expression of DPP4 has been demonstrated as high enzymatic activity in the rat kidney. Besides incretin, several candidates of DPP4 in the kidney have been identified, which may participate in the inflammatory response process. DPP4 was originally described as a $\mathrm{T}$ cell differentiation/activation marker ${ }^{31}$ and DPP4 may affect leukocyte migration. ${ }^{32}$ DPP4 shows nonenzymatic actions such as binding to the adjacent membrane proteins, as well as extracellular matrix proteins, to exert adhesion moleculelike functions. It is suggested that DPP4 inhibition may inhibit macrophage infiltration through GLP-1-independent pathways.

There is evidence to support the mutual activation of MCs and macrophages in cardiovascular and metabolic diseases. MCs activate macrophages by releasing inflammatory cytokines, enzymes, growth factors, and so on, and macrophages seem to activate MCs in airway allergic 
responses. ${ }^{33}$ Both MCs and macrophages were reduced after incretin-based treatments, maybe partly due to attenuated interactions.

Secretory granules of MCs and M1 macrophages contain TNF- $\alpha$, IL- 6 , and TGF- $\beta 1$. TNF- $\alpha$ and IL- 6 could promote inflammatory cell recruitment and activation. After incretin-based therapies, decreased expressions of TNF- $\alpha$ and IL-6 in renal cortex may be mainly attributed to decreased local M1 macrophage and MC infiltration, which in turn reduced M1 macrophage and MC infiltration. The vicious circle was prevented with incretin-based therapies in the present study.

\section{Conclusion}

Incretin-based treatments could alleviate HFD-induced ORG partly through the systemic insulin sensitivity improvement and the attenuated local inflammation, mainly by the decrease of MC and M1 macrophage infiltration and reduction of TNF- $\alpha$ and IL-6.

\section{Acknowledgments}

The authors thank Hong Zhang from the Department of Endocrinology of Peking University First Hospital for material support; technicians from the Laboratory Animal Center for animal operational assistance, including Shenshen Kong, Guosheng Yang, Yinghong Tao, Yuanyuan $\mathrm{Ma}$, and Yongyan $\mathrm{Hu}$; and technicians from the Electron Microscopy Room for observational assistance, including Suxia Wang, Lijun Chai, Yali Ren, and Ming Cheng.

\section{Author Disclosure Statement}

No competing financial interests exist.

\section{References}

1. Wang $\mathrm{Y}$, Chen $\mathrm{X}$, Song $\mathrm{Y}$, et al. Association between obesity and kidney disease: A systematic review and metaanalysis. Kidney Int 2008;73:19-33.

2. Maric-Bilkan C. Obesity and diabetic kidney disease. Med Clin North Am 2013;97:59-74.

3. Griffin KA, Kramer H, Bidani AK. Adverse renal consequences of obesity. Am J Physiol Renal Physiol 2008;294:F685-F696.

4. Ritz E, Koleganova N, Piecha G. Is there an obesitymetabolic syndrome related glomerulopathy?. Curr Opin Nephrol Hypertens 2011;20:44-49.

5. Tran HA. Obesity-related glomerulopathy. J Clin Endocrinol Metab 2004;89:6358.

6. Chalmers L, Kaskel FJ, Bamgbola O. The role of obesity and its bioclinical correlates in the progression of chronic kidney disease. Adv Chronic Kidney Dis 2006;13: 352-364.

7. Mantovani A, Sica A, Sozzani S, et al. The chemokine system in diverse forms of macrophage activation and polarization. Trends Immunol 2004;25:677-686.

8. Gordon S. Alternative activation of macrophages. Nat Rev Immunol 2003;3:23-35.

9. Gordon S, Taylor PR. Monocyte and macrophage heterogeneity. Nat Rev Immunol 2005;5:953-964.
10. Eddy AA. Mast cells find their way to the kidney. Kidney Int 2001;60:375-377.

11. Stevens RL, Austen KF. Recent advances in the cellular and molecular-biology of mast-cells. Immunol Today 1989; 10:381-386.

12. Raible DG, Schulman ES, Dimuzio J, et al. Mast-cell mediators prostaglandin-D2 and histamine activate human eosinophils. J Immunol 1992;148:3536-3542.

13. Silver RB, Reid AC, Mackins CJ, et al. Mast cells: A unique source of renin. Proc Natl Acad Sci U S A 2004;101:1360713612.

14. Metcalfe DD. Mast cells and mastocytosis. Blood 2008; 112:946-956.

15. Lee YS, Jun HS. Anti-inflammatory effects of GLP-1-based therapies beyond glucose control. Mediators Inflamm 2016;2016:3094642.

16. Wang X, Chen H, Zhang M, et al. Roles of mast cells and monocyte chemoattractant protein-1 in the renal injury of obesity-related glomerulopathy. Am J Med Sci 2013;346: 295-301.

17. Reena, Kaur T, Kaur A, et al. Mast cell stabilizers obviate high fat diet-induced renal dysfunction in rats. Eur J Pharmacol 2016;777:96-103.

18. Liu WJ, Xie SH, Liu YN, et al. Dipeptidyl peptidase IV inhibitor attenuates kidney injury in streptozotocininduced diabetic rats. J Pharmacol Exp Ther 2012;340: $248-255$.

19. Glorie LL, Verhulst A, Matheeussen V, et al. DPP4 inhibition improves functional outcome after renal ischemiareperfusion injury. Am J Physiol Renal Physiol 2012;303: F681-F688.

20. Buse JB, Comm LS. Liraglutide and cardiovascular outcomes in type 2 diabetes reply. $N$ Engl J Med 2016;375: 1798-1799.

21. Zhang ZH, Wei F, Vaziri ND, et al. Metabolomics insights into chronic kidney disease and modulatory effect of rhubarb against tubulointerstitial fibrosis. Sci Rep 2015;5: 14472.

22. Hiromura K, Kurosawa M, Yano S, et al. Tubulointerstitial mast cell infiltration in glomerulonephritis. Am J Kidney Dis 1998;32:593-599.

23. Morales E, Valero MA, Leon M, et al. Beneficial effects of weight loss in overweight patients with chronic proteinuric nephropathies. Am J Kidney Dis 2003;41: 319-327.

24. De Cosmo S, Menzaghi C, Prudente S, et al. Role of insulin resistance in kidney dysfunction: Insights into the mechanism and epidemiological evidence. Nephrol Dial Transpl 2013;28:29-36.

25. Nakae S, Suto H, Kakurai M, et al. Mast cells enhance T cell activation: Importance of mast cell-derived TNF. Proc Natl Acad Sci U S A 2005;102:6467-6472.

26. Wang J, Shi GP. Mast cell stabilization: Novel medication for obesity and diabetes. Diabetes Metab Res Rev 2011; 27:919-924.

27. Gschwandtner M, Paulitschke V, Mildner M, et al. Proteome analysis identifies L1CAM/CD171 and DPP4/CD26 as novel markers of human skin mast cells. Allergy 2017; 72:85-97.

28. Neuhofer A, Zeyda M, Mascher D, et al. Impaired local production of proresolving lipid mediators in obesity and 17-HDHA as a potential treatment for obesity-associated inflammation. Diabetes 2013;62:1945-1956.

29. Ye YM, Qian JQ, Castillo AC, et al. Phosphodiesterase-3 inhibition augments the myocardial infarct size-limiting 
effects of exenatide in mice with type 2 diabetes. $A m$ J Physiol Heart Circ Physiol 2013;304:H131-H141.

30. Higashijima Y, Tanaka T, Yamaguchi J, et al. Antiinflammatory role of DPP-4 inhibitors in a nondiabetic model of glomerular injury. Am J Physiol Renal Physiol 2015;308:F878-F887.

31. Reinhold D, Goihl A, Wrenger S, et al. Role of dipeptidyl peptidase IV (DP IV)-like enzymes in T lymphocyte activation: Investigations in DP IV/CD26-knockout mice. Clin Chem Lab Med 2009;47:268-274.

32. Schmiedl A, Krainski J, Schwichtenhovel F, et al. Reduced airway inflammation in CD26/DPP4-deficient F344 rats is associated with altered recruitment patterns of regulatory $\mathrm{T}$ cells and expression of pulmonary surfactant proteins. Clin Exp Allergy 2010;40:1794-1808.
33. Shiraishi D, Fujiwara Y, Komohara Y, et al. Glucagon-like peptide-1 (GLP-1) induces M2 polarization of human macrophages via STAT3 activation. Biochem Biophys Res Commun 2012;425:304-308.

Address correspondence to: Geheng Yuan, PhD Department of Endocrinology Peking University First Hospital

No. 8 Xishiku Street Xicheng District Beijing 100034

People's Republic of China

E-mail:139197109@qq.com 